\title{
Récit de tournage : le documentaire comme méthode de recherche-action
}

\section{The documentary as an action research method: story of a film shoot}

\section{Historia de una filmación: el documental como método de investigación-acción}

\section{François-Xavier Charlebois}

Volume 47, numéro 1, printemps 2019

Entre décrochage(s) et raccrochage(s) scolaires : paroles de jeunes et mises en récit

URI : https://id.erudit.org/iderudit/1060845ar

DOI : https://doi.org/10.7202/1060845ar

\section{Aller au sommaire du numéro}

\section{Éditeur(s)}

Association canadienne d'éducation de langue française

ISSN

1916-8659 (numérique)

Découvrir la revue

Citer cet article

Charlebois, F.-X. (2019). Récit de tournage : le documentaire comme méthode de recherche-action. Éducation et francophonie, 47(1), 15-30.

https://doi.org/10.7202/1060845ar
Résumé de l'article

La problématique sociale du raccrochage scolaire fait l'objet de peu d'attention de la part des chercheurs (Voyer, Potvin et Bourdon, 2014). Les recherches menées de l'intérieur à partir de la parole des acteurs concernés par la problématique sont d'autant plus rares. En 2012, le réseau de recherche-action PARcours, coordonné par Danielle Desmarais, professeure à l'École de travail social de l'Université du Québec à Montréal, a produit un long métrage documentaire réalisé par Jonathan Durand. Ce film, intitulé L'école pour moi, consigne les récits de vie de 14 jeunes provenant de quatre pays (Canada [Québec], France, Belgique et Espagne). Cet article présente les prémisses épistémologiques de notre production et propose une réflexion sur le potentiel du documentaire comme outil de recherche-action. L’usage de la vidéo révèle un fort potentiel de développement de réflexivité pour les jeunes ayant travaillé à mettre leur vie en récit devant la caméra. Deuxièmement, le film constitue un outil important pour la formation de futurs pédagogues et pour l'accompagnement de jeunes en situation de raccrochage scolaire. Troisièmement, la méthode du go-along que nous avons employée révèle, pour les scientifiques, un fort potentiel en ce qui a trait à la production de données originales et complémentaires à des méthodes d'entretien biographique formel. Ces méthodes et les retombées observées rendent compte du dynamisme de la recherche-action dans les disciplines pratiques comme le travail social et l'éducation.
Tous droits réservés (C) Association canadienne d'éducation de langue française, 2019
Ce document est protége par la loi sur le droit d'auteur. L'utilisation des services d’Érudit (y compris la reproduction) est assujettie à sa politique d'utilisation que vous pouvez consulter en ligne.

https://apropos.erudit.org/fr/usagers/politique-dutilisation/ 


\section{Récit de tournage: le documentaire comme méthode de recherche-action}

François-Xavier CHARLEBOIS

Université de Montréal, Québec, Canada

\section{RÉSUMÉ}

La problématique sociale du raccrochage scolaire fait l'objet de peu d'attention de la part des chercheurs (Voyer, Potvin et Bourdon, 2014). Les recherches menées de l'intérieur à partir de la parole des acteurs concernés par la problématique sont d'autant plus rares. En 2012, le réseau de recherche-action PARcours, coordonné par Danielle Desmarais, professeure à l'École de travail social de l'Université du Québec à Montréal, a produit un long métrage documentaire réalisé par Jonathan Durand. Ce film, intitulé L'école pour moi, consigne les récits de vie de 14 jeunes provenant de quatre pays (Canada [Québec], France, Belgique et Espagne). Cet article présente les prémisses épistémologiques de notre production et propose une réflexion sur le potentiel du documentaire comme outil de recherche-action. L'usage de la vidéo révèle un fort potentiel de développement de réflexivité pour les jeunes ayant travaillé à mettre leur vie en récit devant la caméra. Deuxièmement, le film constitue un outil important pour la formation de futurs pédagogues et pour l'accompagnement de jeunes en situation de raccrochage scolaire. Troisièmement, la méthode du go-along que nous avons employée révèle, pour les scientifiques, un fort potentiel en ce qui a trait à la production de données originales et complémentaires à des méthodes d'entretien biographique formel. Ces méthodes et les retombées observées rendent compte du dynamisme de la recherche-action dans les disciplines pratiques comme le travail social et l'éducation. 


\section{ABSTRACT}

\section{The documentary as an action research method: story of a film shoot}

The social problem of re-entering the school system has received little attention from researchers (Voyer, Potvin and Bourdon, 2014). Research examining the testimonies of those concerned is all the more rare. In 2012, the PARcours action research network coordinated by Danielle Desmarais, professor at the University of Quebec in Montreal School of Social Work, produced a documentary feature film directed by Jonathan Durand. «The School for Me» records the life stories of 14 young people from four countries (Canada [Quebec], France, Belgium and Spain). This article presents the epistemological premises of our production and proposes a reflection on the potential of the documentary as an action research tool: (1) The use of video has a strong potential for developing reflexive thinking in young people who have worked to put their lives in narrative form in front of a camera; (2) The film is an important tool for the training of future educators and for helping young people in their process of returning to school; (3) The go-along method used in this study shows great potential for scientists in terms of producing raw or complementary data for formal biographical interview methods. These methods and their observed benefits reflect the dynamism of action research in practical disciplines such as social work and education.

\section{RESUMEN}

\section{Historia de una filmación: el documental como método de investigación-acción}

La problemática social de la deserción escolar ha sido un objeto poco atendido por los investigadores (Voyer, Potvin y Bourdon, 2014). Las investigaciones realizadas al interior a partir de la palabra de los actores concernidos por la problemática son aun más raras. En 2012 la red de investigación-acción PARcours, bajo la coordinación de Danielle Desmarais, profesora de la Escuela de trabajo social de la Universidad de Quebec en Montreal, produjo un largometraje documental realizado por Jonathan Durand. Esta película, titulada L'ecole pour moi, consigna las historias de vida de 14 jóvenes provenientes de cuatro países (Canadá [Quebec], Francia, Bélgica y España). Este artículo presenta las premisas epistemológicas de nuestra producción y propone una reflexión sobre el potencial del documental como herramienta de investigación-acción. El empleo del video muestra un fuerte potencial para el desarrollo de la reflexividad entre los jóvenes que trabajaron en la filmación de sus historias de vida. Además, la película constituye una herramienta importante para la formación de futuros pedagogos y para el acompañamiento de jóvenes en situación de reinserción escolar. Finalmente, el método go-along que empleamos muestra a los científicos un fuerte potencial en lo que se refiere a la producción de datos originales y complementarios de los métodos de entrevista biográfica formal. Dichos métodos y resultados 
observados reflejan el dinamismo de la investigación-acción en las disciplinas prácticas como el trabajo social y la educación.

\section{INTRODUCTION}

Au printemps 2012, une équipe de tournage mandatée par le réseau de recherche-action PARcours ${ }^{1}$ lançait les opérations pour produire le film documentaire L'école pour moi. Parcours de raccrochage scolaire (Desmarais, Durand et Charlebois, 2013) sur les parcours de vie de 14 jeunes adultes en situation de raccrochage scolaire dans quatre pays: le Canada, la France, l'Espagne et la Belgique. L'équipe avait reçu le mandat d'opérationnaliser le tournage et le montage du film en s'appuyant sur une méthodologie alliant art cinématographique et sciences humaines. L'objectif de cette production était de créer un format original permettant de donner la voix et de comprendre la parole de ceux qui sont concernés au premier chef par le phénomène du décrochage et du raccrochage scolaires: les jeunes adultes eux-mêmes. Le film a été réalisé avec les partenaires internationaux du réseau PARcours afin de connaître différents dispositifs de raccrochage scolaire, et de cerner les différences et les similitudes dans les parcours des jeunes en rupture avec l'école. J'ai participé à toutes les étapes de la production de ce film à titre de professionnel de recherche. Dans cet article, je présenterai les référents théoriques sur lesquels nous nous sommes appuyés pour créer le dispositif méthodologique employé pour produire le film. Puis, en m’appuyant sur des données filmiques (des scènes du film) et empiriques (recueillies dans le cadre de la phase de diffusion du film), je présenterai les retombées de cette méthodologie à trois niveaux: pour les personnes participantes, pour la production scientifique et pour les publics du documentaire. Nous verrons que la parole des jeunes est une source de connaissance indispensable pour comprendre l'expérience du décrochage scolaire et les dispositifs de raccrochage scolaire.

\section{UN RÉSEAU DE RECHERCHE-ACTION PRÉCONISANT L'APPROCHE CLINIQUE EN SCIENCES HUMAINES}

En 2010, à l'instigation de Danielle Desmarais, le réseau de recherche-action PARcours a été créé par des partenaires du milieu de la recherche et de l'intervention dans le domaine du raccrochage scolaire. Ces partenaires provenaient de pays et d'horizons disciplinaires variés (éducation spécialisée, travail social, organisme communautaire

1. Danielle Desmarais, anthropologue et professeure à l'École de travail social de I'Université du Québec à Montréal (UQAM), a assuré la coordination scientifique du réseau PARcours (Pratique d'accompagnement du raccrochage scolaire) de 2010 à 2017. 
d'éducation populaire, commissions scolaires ${ }^{2}$ ). Le réseau PARcours visait deux objectifs précis: comprendre les parcours de vie des jeunes adultes de 16 à 20 ans en situation de raccrochage scolaire et étudier les pratiques d'accompagnement éducatif déployées en milieu scolaire et communautaire.

Un autre objectif, celui-ci plus fondamental et relevant d'une approche anthropologique, était de comprendre les histoires de vie de jeunes adultes dans une perspective globale. Le phénomène du décrochage/raccrochage à l'entrée dans l'âge adulte est un «fait social total», selon Desmarais (2013a), car il interpelle toutes les dimensions de l'humain (identitaire, symbolique, sociale, économique, etc.) (Mauss, 1923, cité dans Dortier, 2004). En ce sens, L'école pour moi offre une représentation globale de l'univers de signification (Geertz, 2003) à l'intérieur duquel vivent de jeunes adultes en situation de raccrochage scolaire.

Ces sensibilités nous ont conduits à adopter l'approche clinique en sciences humaines (Sévigny, 1993) pour appréhender nos objets de recherche. En cohérence avec une approche anthropologique, cette posture théorique permet de saisir la réalité globale des jeunes adultes en situation de raccrochage scolaire. L'approche clinique en sciences humaines est donc intrinsèquement interdisciplinaire (Sévigny, 1993). Elle vise à comprendre le sens du vécu d'un individu, d'un groupe ou d'une collectivité en articulant les dimensions psychiques et sociales (de Gaulejac, 2009). Desmarais (2012) propose un découpage de la réalité sociale à trois niveaux (microsocial, mésosocial et macrosocial) pour connaître, en plus des déterminants psychiques et macrosociaux, les spécificités des groupes, les organisations ainsi que les instances de socialisation comme les amis et la famille qui ont façonné l'individu dans son histoire. De plus, l'approche clinique en sciences humaines est très complémentaire de la psychologie historico-culturelle de Vygotsky (Clot, 2002). Comme la psychologie du développement élaborée par Vygotsky, l'approche clinique en sciences humaines postule que la psychologie d'un sujet constitue une conséquence irréductible et imprévisible du social (Clot, 2002; de Gaulejac, 2009; Sève, 2008; Vygotsky, 1997). L'adoption d'une approche biographique permet de comprendre les phénomènes sociaux, comme le décrochage et le raccrochage scolaires, dans leur face subjective en prenant en compte l'histoire individuelle et l'ensemble des rapports sociaux participant de l'expérience vécue.

C'est en adoptant cette approche que le réseau PARcours a réalisé le long métrage documentaire L'école pour moi diffusé en 2013. Ce film a été réalisé en cohérence avec les principes de la recherche-action (Rhéaume, 1982) avec l'ensemble des partenaires impliqués dans le tournage. Les partenaires ont élaboré les paramètres de la production et ont participé à la catégorisation des chapitres du film. Ils ont aussi facilité le recrutement des "personnages»: 14 jeunes adultes en situation de raccrochage scolaire dans des dispositifs d'accompagnement et des pays différents. Pour participer à

2. Le territoire québécois est divisé en 72 commissions scolaires dont le mandat est de promouvoir l'éducation et d'administrer les ressources scolaires. 
la recherche, les jeunes devaient être âgés de 16 à 25 ans, avoir décroché du secteur des jeunes et être inscrits dans un dispositif de raccrochage scolaire communautaire ou offert par un établissement d'enseignement public. L'approche globale préconisée par l'équipe de tournage permet d'entendre, à l'écoute du film, la parole des jeunes sur des dimensions significatives de leur parcours de vie et de leurs expériences scolaires éprouvantes. La deuxième moitié du documentaire présente le point de vue des jeunes adultes sur les organismes et les intervenants qui les accompagnent.

\section{UN FILM DANS LEQUEL ART ET SCIENCES HUMAINES SE RENCONTRENT}

Pour offrir cette représentation, nous avons décidé de juxtaposer des méthodes artistiques et scientifiques. Cette méthode n'est pas classique et est absente des ouvrages de méthodologie de recherche en sciences humaines. Dès lors, les documentaires produits par les chercheurs n'obtiennent pas la même reconnaissance que les publications écrites (Friedmann, 2006). Certains se sont chargés de nous le rappeler lors de la diffusion du documentaire dans les milieux de pratique et de recherche. "Il faut distinguer une production scientifique de ce qui relève de l'intervention", nous a-t-on dit. Or, les chercheurs qui se réclament de l'approche clinique en sciences humaines cherchent précisément, dans la foulée de Kurt Lewin ${ }^{3}$, à remettre en question les frontières établies entre science et pratiques. Néanmoins, la question se pose: quel statut épistémologique a l'image?

Selon Friedmann (2006), l'image comme outil de connaissance est refusée depuis longtemps dans plusieurs traditions des sciences humaines. Déjà, Platon voyait dans l'image l'illusion d'une connaissance du monde. Encore aujourd'hui, les chercheurs qui ont recours à l'image pour transmettre des connaissances sont accusés d'utiliser des outils trop équivoques et polysémiques. «La nécessité d'interpréter un texte n'est pas tout à fait du même type que celle d'interpréter une image qui, contrairement au texte, ne dispose ni de dictionnaire ni de grammaire pour être déchiffrée et comprise» (Péquignot, 2006). Pour adhérer à cette citation, encore faut-il être en faveur d'une position objectiviste du langage selon laquelle les mots reflètent la pensée de la personne qui s'exprime et les définitions contenues dans le dictionnaire. Une autre position, plus dynamique et empruntée à Wittgenstein, postule que la compréhension des mots relève plutôt d'un accord implicite entre les personnes impliquées dans une situation unique (Alvesson et Sköldberg, 2009). Dès lors, il ne faut pas se référer au dictionnaire pour comprendre les mots mobilisés par les personnes pour exprimer leur pensée. Il faut plutôt analyser l'usage singulier qu'une personne fait du mot (Younes, 2016). Cette signification apparaît dans les règles de l'interaction et de la situation dans laquelle communique la personne (Huot, 2013). En ce sens, les situations vidéographiées captées selon une approche clinique en sciences humaines (donc globale) permettent d'observer une personne dans ses rapports sociaux. Si on

3. Kurt Lewin est l'auteur de cette fameuse citation: «Il n'y a rien de plus pratique qu'une bonne théorie» (Vallerand, 1994).

VOLUME XLVII : 1 - printemps 2019 
adhère à la position de Wittgenstein, ces images permettent, contrairement à la position exposée ci-dessus, de mieux comprendre le sens que les personnes donnent aux situations vécues. Mais l'écriture permet-elle de rendre compte aussi globalement du sens des énoncés produits?

L'équipe de recherche a produit le film en adhérant à l'idée que le cinéma documentaire n'a pas la même fonction que l'outil du langage écrit. Nous pensions plutôt, à l'instar de Laplantine, que la caméra donne une «leçon d'écriture» (Laplantine, 1996). Il n'est pas aisé de rendre compte par écrit de la densité des phénomènes contenus dans une image. La particularité de l'image est de contenir une grande richesse interprétative. Cette richesse permet de comprendre (et non d'expliquer) les faits sociaux de façon phénoménologique, c'est-à-dire à travers l'expérience, le vécu capté par la caméra. Dans le chapitre du film qui traite de la concentration à l'école une épreuve qu'ont eu à traverser plusieurs personnes participantes -, Bobby raconte les situations qu'il vivait à l'école lorsqu'il était confronté à l'anxiété et à l'échec. Son récit évoque avec puissance le sentiment de honte qu'il ressentait dans le milieu scolaire. Simultanément, on observe son corps en train de maîtriser la violence que lui fait ressentir la répétition psychique des souvenirs de l'expérience scolaire. Certes, ces images sont équivoques. Il en revient aux publics du documentaire de débattre du sens donné aux communications comme les silences, les postures, les hésitations, etc. Néanmoins, les données filmiques rendent mieux compte du langage corporel que les notes recueillies dans un journal de bord lors du terrain. Du moins, elles en rendent davantage compte matériellement, bien que l'interprétation demeure équivoque, inévitablement.

De plus, contrairement aux critiques adressées à l'usage du documentaire en sciences humaines, les émotions suscitées par le visionnement d'images ne constituent pas une entrave à la distance intellectuelle nécessaire au travail scientifique ni une forme de contagion émotionnelle des publics. Les réactions de l'observateur, en d'autres mots, le contre-transfert (Devereux, 2012), sont inévitables, et seul un travail réflexif permet de déceler ces «déformations» dans l'interprétation qu'il fait des comportements des sujets à l'étude.

La distance consiste précisément à prendre conscience des émotions provoquées par l'écoute des récits afin de les distinguer du sens que les participants donnent à leur propre vécu. En ce sens, nous verrons, dans la prochaine section, que la méthode d'organisation des données que nous avons employée dans l'analyse des données filmiques est rigoureuse. Elle vise à prendre conscience du phénomène de contre-transfert dans la relation avec l'autre et à l'expliciter (Devereux, 2012). À cet égard, les choix esthétiques du film témoignent de notre sensibilité pour le vécu des jeunes adultes.

Les personnes qui mènent des recherches avec une approche clinique en sciences humaines assument, dans la foulée de la sociologie de Weber, de rompre avec l'idéal 
de neutralité (Hanique, 2012). En ce sens, la facture visuelle du film est en soi un parti pris pour la parole des jeunes comme source de vérité sur leur réalité. Les images captées et choisies lors du montage cherchent à les valoriser physiquement et psychologiquement. Les publics auprès desquels nous diffusons le film nous l'ont confirmé plus d'une fois: nous avons réussi à produire un «beau» film, c'est-à-dire un film "plaisant à regarder» (Dufour, 2015). Notre objectif était que le plaisir esthétique soit lié à la beauté des images, et ce, de manière à reconnaître la charge sensible des paroles des personnes participantes. Nous ne recherchions pas seulement la finalité du plaisir esthétique. Les images jugées belles ont le pouvoir de solliciter l'empathie, voire l'affection à l'écoute des récits des personnages du film. L'écriture scientifique peut, doit être sensible lorsqu'elle rend compte d'un vécu souffrant. À cet égard, l'usage de la caméra dans la production scientifique dispose de moyens sonores et visuels très efficaces pour représenter la sensibilité du vécu. Puisque l'image est riche de sens, la combinaison d'une démarche artistique et scientifique sert bien l'approche clinique en sciences humaines qui consiste à comprendre, par l'interprétation, le vécu des personnes participant à la recherche, et ce, avec sensibilité. Encore faut-il apprécier la valeur épistémologique du récit, reconnaître le caractère sensible du matériau biographique et accorder de la crédibilité à l'interprétation comme activité scientifique.

\section{APPUIS ÉPISTÉMOLOGIQUES ET MÉTHODOLOGIQUES}

Les prémisses épistémologiques du film sont, à l'instar de la pensée de Ricœur, herméneutiques et phénoménologiques. Humaniste, Ricœur (1990) postule que la liberté de l'individu réside dans son pouvoir d'action et, de surcroît, dans son pouvoir de donner un sens à ses actions, a posteriori. Cet individu à la recherche du sens de ses actions a recours au récit de soi. Puisque la description d'une situation succède à l'action, elle entre inévitablement en décalage interprétatif avec celle-ci. Ce décalage entre le récit et l'action est un problème épistémologique d'envergure. Comment le récit peut-il rendre compte des actions posées? Selon Ricœur, le récit interprétatif est l'inévitable et l'équivoque accès à une compréhension empirique. Le récit est un besoin humain, puisqu'il est source de conscience de soi (conscience dynamique et faite d'illusion, notamment, mais conscience tout de même) et du monde dans lequel nous vivons. De plus, pour les jeunes adultes, la mise en récit revêt une importance cruciale à un âge marqué par la quête de soi (Erikson, 2011).

Étant donné les contraintes inhérentes au terrain de recherche, nous disposions d'une journée avec chaque jeune adulte pour recueillir son récit. Nous avons adopté une méthodologie de recherche combinant deux approches: la méthode go-along et l'approche biographique. La méthode go-along, ou méthode itinérante, est «une méthode hybride entre l'observation participante et l'entrevue où le chercheur accompagne une personne dans sa routine en lui posant des questions à partir d'un schéma d'entretien plus ou moins prévisible» (Kusenbach, 2012). La marche est une 
expérience multisensorielle riche sur le plan phénoménologique. C'est une activité qui permet de s'approprier un espace et de lui donner un sens. L'après-midi était consacré à un entretien biographique vidéographié durant lequel une personne de l'équipe de recherche invitait chaque jeune à mettre en récit son histoire de vie en se centrant spécifiquement sur ses liens avec l'école, la famille, les amis et les enseignants, ainsi que sur la société dans laquelle il vit et dans laquelle il se projette.

\section{APPORTS DU DISPOSITIF MÉTHODOLOGIQUE}

La méthodologie de recherche-action que nous avons employée a eu des retombées chez les trois acteurs principaux du documentaire: les jeunes adultes, les chercheurs et les publics.

\section{Pour les jeunes: il favorise la mise en récit}

L'histoire de vie réside dans le rapport intime aux personnes et aux événements significatifs qui sont constitutifs de l'identité narrative (Ricœur, 1990). Pour les jeunes adultes en situation de raccrochage scolaire dont les parcours de vie sont ponctués d'épreuves sociales à différents niveaux de réalité, la narration représente un défi (Charlebois, 2018b). Le langage est un outil de la pensée permettant d'exprimer les contenus de la conscience (Vygotsky, 1997). Or, la parole des jeunes adultes est potentiellement inhibée par la souffrance. En effet, les pensées provoquent des expériences psychiques dont la personne cherche à se défendre afin d'éviter leur répétition (Nasio, 2016). Ces mécanismes psychiques de refoulement ne sont pas seulement des obstacles thérapeutiques, ils constituent aussi des défis à surmonter en situation de recherche, et donc en situation de collecte de données.

À cet égard, la méthode go-along s'est avérée productive. Les méthodes itinérantes sont plus "démocratiques» que plusieurs méthodes de collecte de données en recherche qualitative. Elles permettent aux personnes interviewées d'avoir du pouvoir sur les paramètres de la recherche (Kusenbach, 2012). Par exemple, dans le cadre du tournage du documentaire, ce sont les jeunes qui nous guidaient lors des itinéraires que nous empruntions avec eux. Ce sont les jeunes qui choisissaient les lieux que nous visitions ensemble dans la ville (le parc où ils passaient du temps lorsqu'ils étaient enfants, leur quartier d'origine, l'école secondaire de laquelle ils ont décroché, le jardin où ils ont travaillé, leur appartement, etc.). Les jeunes revivaient des situations passées en les revisitant. Une des séquences du documentaire met en scène Christiane dans l'école secondaire qu'elle a fréquentée par le passé. Elle y était victime d'intimidation par ses pairs. La visite de l'école et des espaces significatifs pour elle a facilité son interprétation des expériences qu'elle y a vécues. Elle indiquait que le territoire de l'école était divisé par des rapports de pouvoir et d'intimidation entre trois groupes de jeunes: «les populaires, les moyens, les rejets». Certains espaces 
appartenaient littéralement aux groupes qui avaient le plus de prestige dans l'école. C'était la parole de Christiane qui construisait l'espace et qui nous permettait de comprendre son rapport à celui-ci. Ces captations permettent aux publics de mieux comprendre le vécu dans la temporalité et dans les espaces, deux éléments indissociables de l'histoire de vie.

Les espaces visités avec les jeunes lors des itinéraires que nous empruntions avec eux étaient marqués par des événements biographiques significatifs. La visite de ceux-ci favorisait la réinterprétation des événements passés. La marche facilitait l'expression libre de la parole, étant donné la proximité avec l'expérience. Cette proximité provoquait des émotions et des attitudes transférables par le récit (Jhala, 2007, cité dans Kusenbach, 2012). Métaphoriquement, il est d'autant plus cohérent de marcher avec les jeunes dans le cadre d'un documentaire dont l'objet est leur parcours de vie.

Marcher avec quelqu'un met en scène deux corps côte à côte. Cette position réduit le caractère intimidant d'un entretien de recherche et le sentiment que certains jeunes peuvent avoir d'être évalués par des chercheurs qui les observent et les analysent (Charlebois, 2018a). Marcher avec les jeunes est aussi cohérent avec la posture de l'approche clinique en sciences humaines. Cette posture repose sur un précaire équilibre entre des rapports inégalitaires et égalitaires coexistants (Charlebois, 2018b). Ces rapports sont inégalitaires, puisque le chercheur se penche sur les réalités d'un groupe en vertu d'un statut de spécialiste. Ils sont simultanément égalitaires, puisque les jeunes adultes sont appréhendés comme des acteurs, des sujets de connaissance. La marche mettait concrètement en mouvement ce rapport égalitaire dans lequel nous formions ensemble une entité qui crée l'espace dialogique (Jones, Bunce, Evans, Gibbs et Ricketts Hein, 2008). Le temps partagé par la marche, une expérience agréable, favorise la création de rapports positifs avec les personnes et d'un lien convivial.

En ajoutant du mouvement dans la collecte de données, les jeunes semblaient également moins intimidés par les moments de silence. J'ai réalisé à ce jour plus d'une trentaine d'entretiens biographiques dans différents projets de recherche, dont ma thèse de doctorat. J'ai observé que certaines personnes cherchent à fuir l'angoisse provoquée par les moments de silence. En situation de silence, le corps devient plus agité, certaines personnes rient nerveusement ou demandent une relance sur un nouveau thème. En situation d'itinérance et de mouvement, il est apparu que ces moments de silence étaient plus supportables, moins angoissants (Charlebois, 2018b). Ainsi, l'expression de mouvement dans la méthodologie de collecte de données autorise les personnes à exprimer du sens avec leur corps, et la caméra permet de le capter. Pour des personnes qui souffrent potentiellement de l'usage du langage, cette occasion est libératrice. Reda, un des personnages du film, nous offre très peu de récits devant la caméra pour exprimer l'impuissance qu'il ressentait dans les situations où il éprouvait des difficultés d'apprentissage. Comparativement aux autres 
participants, ses réponses sont courtes, retenues. Pourtant, son corps s'exprime et nous permet d'interpréter son langage intérieur, voire de l'écouter.

\section{Pour les chercheurs: il permet une compréhension des parcours des jeunes adultes}

Le film que nous avons réalisé vise à comprendre la réalité des jeunes adultes en situation de raccrochage scolaire «de l'intérieur» (Pires, 1997), c'est-à-dire que l'objet de la recherche était le point de vue des jeunes sur le monde dans lequel ils vivent. Comment comprendre ce point de vue? Dans la production d'un documentaire, trois groupes d'acteurs sont actifs dans l'interprétation des récits: les jeunes, les producteurs du documentaire et les publics. Nous avons donc fait le choix de faire de la parole des jeunes le matériau exclusif du contenu du documentaire. Sensibles au point de vue selon lequel les récits individuels sont des contenus culturels polysémiques, nous voulions les laisser exister dans leur richesse interprétative. La présence de propos d'experts dans le documentaire aurait potentiellement réduit cette richesse.

Bien que nous ayons choisi de faire du documentaire un espace réservé à la parole des jeunes, l'intervention de l'équipe de recherche dans la production du film n'est pas neutre pour autant. Le schéma d'entretien biographique et la grille d'observation participante ont été élaborés à la suite de la recension des écrits et de la consolidation des cycles de recherche antérieurs du réseau PARcours. Néanmoins, notre médiation est la plus rigoureuse et discrète possible afin que la production reflète le sens que les jeunes donnent singulièrement à leur parcours de vie. Pour ce faire, nous avons employé les méthodes d'analyse thématique de contenus. Les 14 récits ont été analysés de manière transversale et abductive

L'approche abductive se distingue des canons de la théorie ancrée (grounded theory) de Glaser et Strauss (2010). Le moment où se rencontrent le cadre théorique d'une recherche et les nouvelles expériences vécues en terrain est abductif, selon Peirce (Peirce, 1931 cité dans Cicourel, 2003). L'abduction est l'analyse, c'est-à-dire l'activité qui consiste à dynamiser les savoirs acquis à l'aide des liens effectués entre les nouvelles données et les recherches antérieures. C'est, selon Alvesson et Sköldberg (2009), une attitude intellectuelle qui permet de mobiliser ses préconceptions pour produire des hypothèses à partir des singularités observées en terrain et de confronter les nouvelles données jusqu'à la production d'une nouvelle compréhension. Ces opérations concilient la déduction et l'induction dans une même activité. Cette méthode a permis de synthétiser soixante-quinze heures de matériau en une heure. Ainsi, les récits que nous avons conservés dans le montage final sont ceux qui sont les plus représentatifs de l'ensemble du corpus de données recueillies. 
Nous avons choisi de procéder d'emblée à une analyse transversale des thèmes. Normalement, une approche biographique nécessite de rendre compte de chaque récit de vie chronologiquement afin d'apprécier la perspective temporelle de l'objet (Desmarais, 2016). Toutefois, la durée du documentaire (une heure) ne nous permettait pas de présenter le développement temporel de chaque récit de vie. L'analyse thématique transversale a tout de même respecté une logique biographique. Ainsi, la catégorisation des récits vidéographiés a permis de présenter les paroles des jeunes en deux parties. La première permet de comprendre la spirale des événements et du vécu ayant mené les jeunes au décrochage scolaire. Cette partie couvre des chapitres traitant de l'enfance, de la concentration à l'école, du passage au secondaire, de l'intimidation, des liens sociaux et de la quête d'autonomie. La deuxième partie permet d'écouter les récits des jeunes sur leurs expériences de raccrochage scolaire. Les propos des personnes permettent de comprendre leur rapport aux différents dispositifs d'accompagnement éducatif dans lesquels nous les rencontrons. Un chapitre traite de leur définition de ce qu'est un bon enseignant.

En recherche, le récit de vie sert des intérêts de production de connaissances. Toutefois, cet exercice est aussi utilisé en formation et en intervention (Desmarais, 2016). Le récit de vie est un outil d'éducation populaire important, puisqu'il permet de prendre conscience des déterminants sociaux et psychiques constitutifs de son histoire de vie, de son vécu (Desmarais, 2003). Même s'il est utilisé dans une démarche de recherche, c'est un outil qui a des retombées développementales pour les participants. La narration est un espace de liberté qui permet d'être auteur de ses propres personnages; en d'autres mots, de s'autodésigner. Le «je suis» est soutenu par les récits du passé. Cette narration invite à un «je peux» : voici ce que le temps a fait de moi et voici ce que je ferai du temps dans l'avenir. Le film constitue un espace de narration permettant aux jeunes de dégager du sens des actions passées et à venir. Ainsi, nous avons réservé les deux derniers chapitres du documentaire aux prises de conscience que font les participantes et les participants au terme de la narration de leur histoire. La narration de son action interpelle toujours une visée éthique (Ricœur, 1990). Les jeunes tirent des leçons de l'écoute de leur propre récit et formulent conséquemment des pistes d'action pour le futur. Ces scènes nous permettent de connaître leurs désirs d'avenir, leurs appréhensions. Jeune femme ayant vécu un processus d'immigration en France, Aïsha nous dit: «J'aimerais bien garder ma liberté». Marc, un jeune homme de Montréal ayant grandi dans un quartier défavorisé, nous confie: «Je veux être riche». Reda, un jeune Bruxellois ayant des difficultés d'apprentissage et de concentration, nous dit: «Je vais réussir! Enfin, j'espère... parce que si je ne réussis pas... ça va être la galère...».

\section{Pour les publics: il permet une compréhension empathique du vécu}

L'équipe de production a tourné, monté et diffusé le film dans l'objectif de fournir un outil de réflexion et de formation aux intervenants et aux formateurs d'éducateurs. 
Comme l'exprime Danielle Desmarais, coordonnatrice scientifique du projet, le contenu du film évite le piège de condamner des groupes, soit les jeunes eux-mêmes ou leurs éducateurs (Desmarais, 2013b). Les propos du public recueillis ${ }^{4}$ à l'occasion du lancement du documentaire à Bruxelles nous confirment que le film joue un rôle de sensibilisation à la "vie réelle ${ }^{5}$ » des jeunes adultes en situation de raccrochage scolaire. L'approche notamment inductive de la collecte de données filmiques est respectueuse des récits intimes des personnes. Les jeunes n'ont pas eu à corroborer nos thèses préalables et à en rendre compte au public. L'écoute des récits des jeunes adultes invite les différents acteurs de l'éducation, sans les blâmer, à penser à des actions permettant de prévenir les différentes ruptures que les jeunes décrivent avec peine devant la caméra.

Les réflexions que les paroles des jeunes suscitent lors des projections dans les universités, les centres de raccrochage scolaire et les organismes communautaires démontrent le potentiel heuristique des récits. Des propos ${ }^{6}$ recueillis à la suite de la première du documentaire qui a eu lieu en mai 2013 indiquent que les publics découvrent la complexité et la variabilité des vécus liés au décrochage scolaire, ainsi que les traces que ce vécu laisse durablement dans l'histoire des jeunes gens. La musique, les images et la trame du documentaire ont été pensées de manière à prédisposer les publics à l'écoute sensible. N'y a-t-il pas, scientifiquement, une grande solidarité entre la posture compréhensive de cette recherche et les capacités d'écoute? C'est par une écoute exempte de jugements et ayant pour seul projet de comprendre l'autre que nous avons accueilli le vécu souffrant des jeunes adultes dont le parcours est constitué de nombreuses épreuves. Le récit a l'effet d'un silence s'il n'est pas écouté (Plummer, 2002). Le caractère esthétique du film vient en soutien à l'acte d'écoute. Les choix esthétiques que nous avons faits lors du montage et de la captation des images sont conformes à la posture compréhensive. Les jeunes sont valorisés par la caméra. Les belles images témoignent de notre parti pris pour la valeur heuristique de leurs récits. La musique évoque les épreuves vécues, la charge sensible de certaines souffrances, une certaine nostalgie de l'enfance ou la réflexivité acquise par la réminiscence des souvenirs.

4. Ces propos ont été recueillis et analysés par Danielle Desmarais et Maurice Cornil dans le cadre d'un bulletin de vulgarisation scientifique du réseau PARcours au mois de septembre 2013 (https://parcours. uqam.ca).

5. Cette expression provient d'un texte signé par Maurice Cornil, directeur d'un service d'accrochage scolaire (SAS) à Bruxelles, paru dans un bulletin de vulgarisation scientifique du réseau PARcours au mois de septembre 2013 (https://parcours.uqam.ca). M. Cornil était partenaire du réseau PARcours. Son équipe utilise le documentaire comme outil de formation, de réflexion et de sensibilisation auprès de divers publics en Europe.

6. Ces propos ont été synthétisés et consignés sur le site de l'équipe de PARcours (https://parcours.uqam. ca) dans la section Vox pop. 


\section{LIMITES DES CHOIX MÉTHODOLOGIQUES}

Il importe de rappeler que les partenaires du réseau PARcours ayant participé à la production du film ont priorisé l'objectif de créer un espace pour que les jeunes adultes puissent se raconter sans la médiation d'experts susceptibles d'instrumentaliser leur parole. L'école est encerclée d'experts de diverses natures dont les discours se confrontent dans l'espace public et médiatique (Boutin, 2012). Nous avons fait le choix alternatif de dépasser ces débats, pourrait-on dire de les dynamiser, en donnant la parole exclusivement aux jeunes adultes. Ce choix méthodologique constitue une force du documentaire. Toutefois, il comporte aussi des limites.

Ce montage a pour conséquence d'éliminer les interactions entre nous et les jeunes adultes. La présence de ces interactions aurait permis aux publics de mieux comprendre les récits des participants. Nous sommes impliqués dans les récits des personnes, ne serait-ce que par le choix des thèmes que nous leur demandons d'approfondir dans leur histoire de vie. Or, le documentaire donne quasiment l'illusion que les experts sont absents de la production. Une des questions adressées à l'équipe de production à la suite de la première projection du documentaire a été la suivante: «Et vous, que pensez-vous du décrochage scolaire?». Devereux n'a-t-il pas cerné cette tendance des chercheurs en sciences humaines à cacher leur subjectivité derrière la parole des personnes interviewées (Devereux, 2012)?

Les chercheurs qui mènent des recherches cliniques en sciences humaines dont l'objectif est de soutenir l'action des praticiens ne peuvent éviter de prendre position sur les changements nécessaires. Toutefois, ils doivent aussi éviter le piège de dire aux intervenants comment travailler (Darré, 1999). Voilà pourquoi nous avons décidé de sélectionner les paroles les plus significatives des jeunes adultes qui ont participé à la production. Plutôt que de formuler des recommandations d'experts, nous proposons aux publics des énoncés riches de sens pour réfléchir avec les acteurs du terrain aux stratégies d'accompagnement éducatif les plus adéquates.

\section{EN GUISE DE CONCLUSION}

L'usage de la caméra apparaît comme un outil pertinent pour mener des recherches situées dans le paradigme interprétativiste. Les images captées permettent de récolter des données riches d'un point de vue phénoménologique. Le caractère esthétique et sensible du visuel favorise l'acte d'écoute à la base de l'approche compréhensive des phénomènes sociaux. Les images, les comportements et les récits sont autant de données pouvant faire l'objet d'une analyse thématique, puis d'une interprétation par les publics du documentaire. Pour les jeunes adultes, la participation à un documentaire a eu des retombées positives en ce qui a trait au développement de réflexivité, de connaissance de soi. Pour l'équipe de recherche, l'interprétation thématique des données constitue une avancée théorique importante pour comprendre 
les convergences et les divergences entre les récits de vie de jeunes adultes en situation de raccrochage scolaire dans divers dispositifs éducatifs et différentes cultures. Puis, pour les publics, les expériences de diffusion indiquent que les récits consignés permettent de recentrer la parole des jeunes adultes dans les débats sur les actions éducatives nécessaires.

\section{Références bibliographiques}

ALVESSON, M. et SKÖLDBERG, K. (2009). Reflexive methodology. New vistas for qualitative research ( $2^{\mathrm{e}}$ éd.). Londres, Royaume-Uni: Sage.

BOUTIN, G. (2012). La guerre des écoles: entre transmission et construction des connaissances. Montréal, Québec: Éditions Nouvelles.

CHARLEBOIS, F.-X. (2018a). Construction identitaire de jeunes adultes en situation de pauvreté dans un contexte de raccrochage scolaire. Voies d'émancipation? (Thèse de doctorat). Université de Montréal.

CHARLEBOIS, F.-X. (2018b). "Terrain» où recherche et accompagnement se confondent dans une approche clinique en sciences humaines. Dans I. Fortier, S. Hamisultane, I. Ruelland, J. Rhéaume et S. Beghdadi (dir.), Clinique en sciences sociales. Sens et pratiques alternatives. Québec, Québec: Presses de l'Université du Québec.

CLOT, Y. (dir.). (2002). Avec Vygotsky. Paris, France: La Dispute.

DARRÉ, J.-P. (1999). La production de connaissance pour l'action. Arguments contre le racisme de l'intelligence. Paris, France: Maison des sciences de l'homme.

DESMARAIS, D. (2003). L'alphabétisation en question. Québec, Québec: Québécor.

DESMARAIS, D. (et coll.). (2012). Contrer le décrochage scolaire par l'accompagnement éducatif. Une étude sur la contribution des organismes communautaires. Québec, Québec: Presses de l'Université du Québec.

DESMARAIS, D. (2013a). École, famille, travail: des parcours de raccrochage scolaire en pointillés. Communication présentée à la Journée Pensons persévérance! organisée par Réussite Montérégie, Candiac. Repéré à https://parcours.uqam.ca/ upload/files/Presentation_DD_PensonsPerseverence_2013.pdf 
DESMARAIS, D. (2013b). Un éventail d'activités autour de «L'école pour moi. Parcours de raccrochage scolaire». Bulletin PARcours, septembre 2013. Repéré à http:// parcours.uqam.ca/upload/files/Bulletins/2013/bulletin_parcours_septembre_2013.pdf

DESMARAIS, D. (2016) L'approche biographique. Dans B. Gauthier (dir.), Recherche sociale. De la problématique à la collecte de données. Québec, Québec: Presses de l'Université du Québec.

DESMARAIS, D. (coordonnatrice scientifique) Durand, J. (réalisateur) et Charlebois, F-X. (professionnel de recherche). (2013). L'école pour moi. Parcours de raccrochage scolaire [Documentaire]. Montréal: Réseau PARcours, UQAM.

DEVEREUX, G. (2012). De l'angoisse à la méthode dans les sciences du comportement ( $2^{\mathrm{e}}$ éd.). Paris, France: Flammarion.

DORTIER (2004). Dictionnaire des sciences humaines. Auxerre: Sciences humaines.

DUFOUR, É. (2015). La valeur d'un film. Philosophie du beau au cinéma. Paris, France: Armand Colin.

ERIKSON, E. H. (1972). Adolescence et crise. La quête de l'identité. Paris, France: Flammarion.

FRIEDMANN,D. (2006).Lefilm, l'écritetlarecherche. Communications, 80,5-18. Repéré à http://www.persee.fr/doc/comm_0588-8018_2006_num_80_1_2370?pageid=t2_18

GAULEJAC, V. de. (2009). Qui est «je»? Sociologie clinique du sujet. Paris, France: Seuil.

GEERTZ, C. (2003). La description dense. Vers une théorie interprétative de la culture. Dans D. Cefaï (dir.), L'enquête de terrain (p.208-233). Paris, France: La Découverte.

GLASER, B. et STRAUSS, A. (2010). La découverte de la théorie ancrée. Paris, France: Armand Colin.

HANIQUE, F. (2012). De la sociologie compréhensive à la sociologie clinique. Dans V. de Gaulejac, F. Hanique et P. Roche (dir.), La sociologie clinique. Enjeux théoriques et méthodologiques (p. 105-130). Toulouse, France: Érès.

HUOT, F. (2013). Interactions et pratiques du théorique en travail social. Dans E. Harper et H. Dorvil (dir.), Le travail social. Théories, méthodologies et pratiques (p. 115-125). Québec, Québec: Presses de l'Université du Québec.

JONES, P., BUNCE, G., EVANS, J., GIBBS, H. et RICKETTS HEIN, J. (2008). Exploring space and place with walking interviews. Journal of Research Practice, 4(2). Repéré à http://jrp.icaap.org/index.php/jrp/article/view/150/161 
KUSENBACH, M. (2012). Mobile methods. Dans S. Delamont (dir.), Handbook of qualitative research in education (p. 252-264). Cheltenham, Royaume-Uni: Edward Elgar Publishing.

LAPLANTINE, F. (1996). La description ethnographique. Paris, France: Armand Colin.

NASIO, J.-D. (2016). Oui, la psychanalyse guérit! Paris, France: Payot.

PÉQUIGNOT, B. (2006). De l'usage des images en sciences sociales. Communications, 80, 41-51. Repéré à https://www.persee.fr/doc/comm_0588-8018_2006_num_80_1_ 2372

PIRES, A. (1997). De quelques enjeux épistémologiques d'une méthodologie générale pour les sciences sociales. Dans J. Poupart, J.-P. Deslauriers, L.-H Groulx, A. Laperrière, R. Mayer et A. Pires (dir.), La recherche qualitative. Enjeux épistémologiques et méthodologiques (p. 3-58). Montréal, Québec: Gaëtan Morin.

PLUMMER, K. (2002). Telling sexual stories. Power, change, and social worlds. Londres, Royaume-Uni: Taylor \& Francis.

RHÉAUME, J. (1982). La recherche-action: un nouveau mode de savoir? Sociologie et sociétés, 14(1), 43-51.

RICEUR, P. (1990). Soi-même comme un autre. Paris, France: Seuil.

SÈVE, L. (2008). Penser avec Marx aujourd'hui. «L'homme»? Paris, France: La Dispute.

SÉVIGNY, R. (1993). L'approche clinique dans les sciences humaines. Dans E. Enriquez (dir.), L'analyse clinique dans les sciences humaines (p.13-28), Montréal, Québec: Saint-Martin.

VALLERAND, R. J. (dir.). (1994). Les fondements de la psychologie sociale. Montréal, Québec: Gaëtan Morin.

VOYER, B., POTVIN, M. et BOURDON, S. (2014). Les transformations et défis actuels de la formation générale des adultes. Revue des sciences de l'éducation, 40 (2), 191-213.

VYGOTSKY, L. (1997). Pensée et langage (4e éd.). Paris, France: La Dispute.

YOUNES, R. (2016). Introduction à Wittgenstein. Paris, France: La Découverte. 